\title{
Transit cosmological models coupled with zero-mass scalar-field with high redshift in higher derivative theory
}

\author{
Archana Dixit ${ }^{1}$, Dinesh Chandra Maurya ${ }^{2}$, Anirudh Pradhan ${ }^{3}$ \\ ${ }^{1,3}$ Department of Mathematics, Institute of Applied Sciences \& Humanities, GLA University, Mathura-281 406, Uttar \\ Pradesh, India \\ ${ }_{1}^{2}$ Department of Mathematics, Faculty of Engineering \& Technology, IASE (Deemed to be University), Sardarshahar-331 \\ 403, Rajsthan, India \\ ${ }^{1}$ E-mail:archana.dixit@gla.ac.in \\ ${ }^{2}$ E-mail:dcmaurya563@gmail.com \\ ${ }^{3}$ E-mail:pradhan.anirudh@gmail.com
}

\begin{abstract}
The present study deals with a flat FRW cosmological model filled with perfect fluid coupled with the zero-mass scalar field in the higher derivative theory of gravity. We have obtained two types of universe models, the first one is the accelerating universe (power-law cosmology) and the second one is the transit phase model (hyperbolic expansion-law). We have obtained various physical and kinematic parameters and discussed them with observationally constrained values of $H_{0}$. The transit redshift value is obtained $z_{t}=0.414$ where the transit model shows signature-flipping and is consistent with recent observations. In our models, the present values of EoS parameter $\omega_{0}$ crosses the cosmological constant value $\omega_{0}=-1$. Also, the present age of the universe is calculated.
\end{abstract}

PACS No.: 98.80.Jk; 95.36.+x; 98.80.-k

Keywords: Flat FRW-universe; Higher derivative theory of gravity; Zero-mass scalar field; Redshift

\section{Introduction}

From the recent supernovae observations ( Garnavich et al. [1, 2], Perlmutter et al. [3, 4, 5], Riess et al. [6] and Schmidt et al. 7]), it was predicted that the expansion rate of the universe is increasing at present. This prediction points towards the presence of something (a kind of repulsive force) in the Universe which is pushing everything farther apart faster than it did fin the early universe. The agent for this repulsive force is attributed to a mysterious entity present in the universe along with the usual matter and radiation. This hypothetical unknown exotic physical entity is termed as dark energy (DE). To know the reason behind this acceleration scenario of the present universe, there were two ways, first one to modify Einstein's field equations and another to add a time-dependent cosmological constant $\Lambda$. In order of modifying Einstein's general theory of reellativity (GR), one is obtained by adding $R^{2}$ factor (square of Ricci scalar curvature) in the Einstein-Hilbert action which generalizes the Einstein's GR theory of gravity. Before the fruitful work of Guth, Starobinsky 8 proposed higher derivative theories of gravity that admit inflation. But the theory come into focus only after the work of Guth [9], in which, he used a temperature phase transition mechanism. The good features of the higher-order theories of gravity are very interesting for a long time. Also, in literature, it is a tradition to get a perturbation theory by adding suitable counter terms, viz to the cosmological term $(\Lambda)$ and the $C^{i j k l} C_{i j k l}, R^{2}$ to the Einstein's action is asymptotically free, well behaved, and formally re-normalizable Stelle [10].

The quantum field theory states a new approach to inflation and finds the dynamical history of the expanding universe through GR. Even if, there is a general belief among researchers that the inflation is a part of cosmological evolution. Because of this, to solve the various problems of inflation by reserving its importance, among the researchers have a continuous contest by presenting different modifications (Linde [11]; Kolb and Turner [12]). Chimento and Jakubi [13] have presented cosmological models with perfect fluid in the context of higher derivative theory of gravity. Even if, a perfect fluid source of matter may explain matter distribution in the universe satisfactorily, but reason behind the accelerating scenario of the universe i.e. also, well known by the dark energy (DE) problem, in literature, can not be explained by perfect fluid, properly. In some literature, it is claim that the dark energy problem can be pledged by adding a higher derivative terms in the transport equation (Muller [14; Hiscock and Salmonson [15]). The theory known by extended irreversible thermodynamics (EIT) 
is a fully relativistic formulation of the theory taking the second derivative terms in the theory, is developed by Israel and Stewart [16, and Pavon et al. [17, Pavon [18. Ratra and Peebles [19] have obtained a new type of cosmological solutions in the context of GR by using transport equation the theory EIT. Pavon and Zimdahl 20] are claimed that a viscous fluid source could be the presence of dark matter that causes the acceleration in expansion of the universe and Zimdahl et al. 21] have claimed to the accelerating expansion due to a effective negative force driven by a cosmic anti-friction force. A consistency of various inflation with an arbitrary potential driving inflation are investigated by Ram [22]. A perfect fluid FRW cosmological model with a minimally coupled scalar field have been investigated by Ellis and Madsen 23. In the case of minimally and non-minimally coupled scalar scalar field, a cosmological solution for the scale factor are obtained with the assumption the kinetic and potential terms for the scalar field are proportional to each other, Barrow and Saich 24, Barrow and Mimoso [25] and Mimoso and Wands [26]. Casana et al. 27] have investigated massless DKP field in a Lyra manifold. Recently, Singh et al. 28, have investigated the cosmological solutions in the presence of an imperfect fluid and zero-mass scalar field in higher derivative theory of gravity. Recently, Maurya and Zia 29] have investigated Brans-Dicke scalar field cosmological model in Lyra's Geometry. Some authors [30-33] have studied and discussed zero-mass scalar field in different contexts.

Motivated by above discussion, we have considered a flat FRW universe filled with perfect fluid coupled with the zero-mass scalar field in higher derivative theory of gravity and obtained a transit phase model. The out lines of the paper as follows: Section 1 is introductory, Section 2 introduces the metric and field equations. Solutions and discussion of results are given in Section 3 and finally conclusions are given in Section 4.

\section{Metric and Field Equations}

To investigate the physical behaviour of the universe, we consider the action principle as

$$
I=\int\left[\frac{1}{2} f(R)+L_{m}\right] \sqrt{-g} d x^{4}
$$

where $f(R)$ is a function of Ricci scalar curvature $R$ and its higher powers, $g$ is the determinant of the metric tensor $g_{i j} . L_{m}$ is matter Lagrangian, in cosmic unit taking $8 \pi G=c=1$. By the variation of action (1) with respect to metric field $g_{i j}$ and scalar field $\phi$ respectively, we get

$$
f^{\prime}(R) R_{i j}-\frac{1}{2} f(R) g_{i j}+f^{\prime \prime}(R)\left(\nabla_{i} \nabla_{j} R-\square R g_{i j}\right)+f^{\prime \prime \prime}(R)\left(\nabla_{i} R \nabla_{j} R-\nabla^{k} R \nabla_{k} R g_{i j}\right)=-T_{i j}
$$

and

$$
\sqrt{-g} \phi_{, i}^{i}=\left(\sqrt{-g} g^{i j} \phi, i\right), j=0
$$

where $\square=g_{i j} \nabla^{i} \nabla^{j}$ and $\nabla_{i}$ is the covariant differential operator, and prime represents the derivative with respect to $R$. The Stress-energy momentum tensor for the fluid coupled with zero-mass scalar-field is given as

$$
T_{i j}=T_{i j}^{F}+T_{i j}^{\phi}
$$

where

$$
T_{i j}^{F}=(\rho+p) u_{i} u_{j}+p g_{i j}
$$

and

$$
T_{i j}^{\phi}=\phi_{, i} \phi_{, j}-\frac{1}{2} g_{i j} \phi_{, k} \phi^{, k}
$$

where $\rho$ is the energy density and $p$ is the isotropic pressure of the fluid. In a co-moving coordinate system, the four-velocity vector $u_{i}$ are satisfies the condition $u_{i} u^{i}=-1 . \phi$ is the zero-mass scalar-field.

Now, we have considered the flat Robertson-Walker spacetime metric, given by

$$
d s^{2}=-d t^{2}+a^{2}(t)\left[d r^{2}+r^{2}\left(d \theta^{2}+\sin ^{2} \theta d \varphi^{2}\right)\right]
$$

where $a(t)$ is the scale factor of the universe. The scalar curvature $R$ and trace $T$ of the energy momentum tensor $T_{i j}$ of the universe are given as respectively

$$
R=-6\left(\dot{H}+2 H^{2}\right)
$$

and

$$
T=-(\rho-3 p)-\dot{\phi}^{2}
$$


where $H=\frac{\dot{a}}{a}$ is the Hubble parameter and an over dot represents a derivative with respect to time. The trace and $(0,0)$ components of (2) are respectively, given by

$$
f^{\prime}(R) R-2 f(R)-3 f^{\prime \prime}(R)(\ddot{R}+3 H \dot{R})-3 f^{\prime \prime \prime}(R) \dot{R}^{2}+T=0
$$

and

$$
f^{\prime}(R) R_{0}^{0}-\frac{1}{2} f(R)-3 f^{\prime \prime}(R) H \dot{R}+T_{0}^{0}=0
$$

Now, since, we have considered a higher derivative theory of gravity defined by $f(R)=R+\alpha R^{2}$. Using Eq. (8) in (10) and (11), we obtain

$$
\dot{H}+2 H^{2}+6 \alpha\left[\dddot{H}+7 H \ddot{H}+4 \dot{H}^{2}+12 H^{2} \dot{H}\right]=-\frac{1}{6} T
$$

and

$$
3 H^{2}+18 \alpha\left[2 H \ddot{H}+\dot{H}^{2}+10 H^{2} \dot{H}\right]=\rho+\frac{\dot{\phi}^{2}}{2}
$$

From Eq. (3), we get

$$
\ddot{\phi}+3 H \dot{\phi}=0
$$

Taking covariant derivative of the field equation (2) and applying energy conservation laws, we obtain

$$
36 \alpha(\ddot{H}+4 H \dot{H})\left(2 \dot{H}+H^{2}\right)=0
$$

and

$$
\dot{\rho}+3 H(\rho+p)=0
$$

where over dot denotes ordinary derivatives with respect to cosmic time ' $t$ '.

\section{Solutions of the field equations and discussion of results}

From redshift relation $\frac{a\left(t_{0}\right)}{a(t)}=1+z$ with $a\left(t_{0}\right)=1$, we have found

$$
\begin{aligned}
& \dot{H}=-(1+z) H H^{\prime} \\
& \ddot{H}=(1+z) H^{2} H^{\prime}+(1+z) H H^{\prime 2}+(1+z)^{2} H^{2} H^{\prime \prime} \\
& \dddot{H}=-(1+z) H^{3} H^{\prime}-4(1+z)^{2} H^{2} H^{\prime 2}-3(1+z)^{2} H^{3} H^{\prime \prime}-(1+z)^{3} H^{2} H^{\prime 3}-4(1+z)^{3} H^{2} H^{\prime} H^{\prime \prime}-(1+z)^{3} H^{3} H^{\prime \prime \prime}
\end{aligned}
$$

where $H^{\prime}=\frac{d H}{d z}$.

Now, integrating Eq. (14), we have obtained the expression for zero-mass scalar field as

$$
\dot{\phi}=\frac{n}{a^{3}}=n(1+z)^{3}
$$

or

$$
\phi=-n \int \frac{(1+z)^{2}}{H} d z
$$

where $n$ is an integrating constant.

Therefore, using these results in Eqs. (12) and (13) we obtain

$$
T=6(1+z) H H^{\prime}-12 H^{2}+36 \alpha(1+z) H^{2}\left[6 H H^{\prime}-4(1+z) H H^{\prime \prime}+(1+z)^{2} H^{\prime 3}+4(1+z)^{2} H^{\prime} H^{\prime \prime}+(1+z)^{2} H H^{\prime \prime \prime}-7 H^{\prime 2}\right]
$$

and

$$
\rho=3 H^{2}+18 \alpha(1+z) H^{2}\left[(3+z) H^{\prime 2}+2(1+z) H H^{\prime \prime}-8 H H^{\prime}\right]-\frac{n^{2}}{2}(1+z)^{6}
$$

If the equation of state $(\operatorname{EoS})$ for the considered fluid is read as $p=\rho \omega$, then EoS parameter $\omega$ is calculated from Eq. (16) as

$$
\omega=-1-\frac{\dot{\rho}}{3 H \rho}
$$

or

$$
2(1+z) H H^{\prime}-n^{2}(1+z)^{6}
$$




$$
\omega=-1+\frac{-12 \alpha(1+z) H\left[2(1+z)\left(2 H H^{\prime 2}-H^{\prime 3}+H^{2} H^{\prime \prime}\right)-(1+z)^{2} H\left(H^{\prime} H^{\prime \prime}+H^{\prime 3}+H H^{\prime \prime \prime}\right)+4 H^{2} H^{\prime}+5 H H^{\prime 2}\right]}{\left[3 H^{2}+18 \alpha(1+z) H^{2}\left[(3+z) H^{\prime 2}+2(1+z) H H^{\prime \prime}-8 H H^{\prime}\right]-\frac{n^{2}}{2}(1+z)^{6}\right]}
$$

Now, from Eq. (15), we have

$$
\begin{aligned}
& \ddot{H}+4 H \dot{H}=0 \quad \text { or } \quad 2 \dot{H}+H^{2}=0 \\
& 2 \dot{H}+H^{2}=0 \quad \Longrightarrow \quad H=\frac{k}{\sqrt{a}}=k(1+z)^{\frac{1}{2}}
\end{aligned}
$$

and

$$
\ddot{H}+4 H \dot{H}=0 \Longrightarrow \dot{H}=\frac{c_{1}}{a^{4}}=c_{1}(1+z)^{4}
$$

where $k$ and $c_{1}$ are arbitrary integrating constant.

Thus, there are two cases to obtain the Hubble parameter and scale-factor which gives two different types of universe model.

\subsection{Accelerating Model: Case-I: when $H=\frac{k}{\sqrt{a}}$}

$$
H=\frac{k}{\sqrt{a}} \Longrightarrow a(t)=\left(\frac{k t+l}{2}\right)^{2}
$$

which shows power-law cosmology and also, in terms of redshift, the Hubble parameter $(H)$ obtained as

$$
H=k(1+z)^{\frac{1}{2}}
$$

It is known that at present $z=0$ putting in Eq. (30), we get the present value of Hubble parameter $H_{0}=k$. Recently, Maurya and Zia [29] estimated the present value of the Hubble parameter $H_{0}=71.27$. Figure 1 represents the variation of Hubble parameter $H(z)$ over redshift $z$. We see that $H(z)$ is an increasing function of redshift $z$ which reveals the expanding nature of the universe.

The deceleration parameter for the model is obtained as a constant negative value $q=-0.5$ that shows that the accelerating scenario of the universe.

Now, Eq. (21) becomes

$$
\phi=\phi_{0}+\frac{2 n}{5 k}\left[1-(1+z)^{\frac{5}{2}}\right]
$$

where $\phi_{0}$ is the present value of scalar field $\phi$. Figures 2(a) and 2(b) represent the variation of scalar field $\phi(z)$ versus redshift $z$.

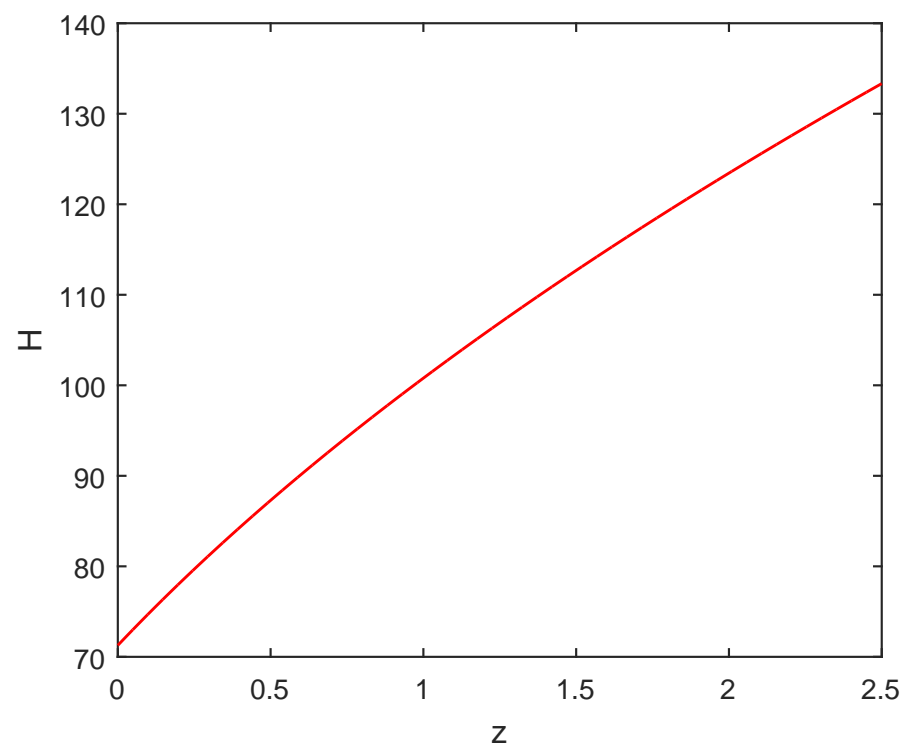

Figure 1: The plot of Hubble parameter $H(z)$ versus redshift $z$ for $H_{0}=71.27$. 

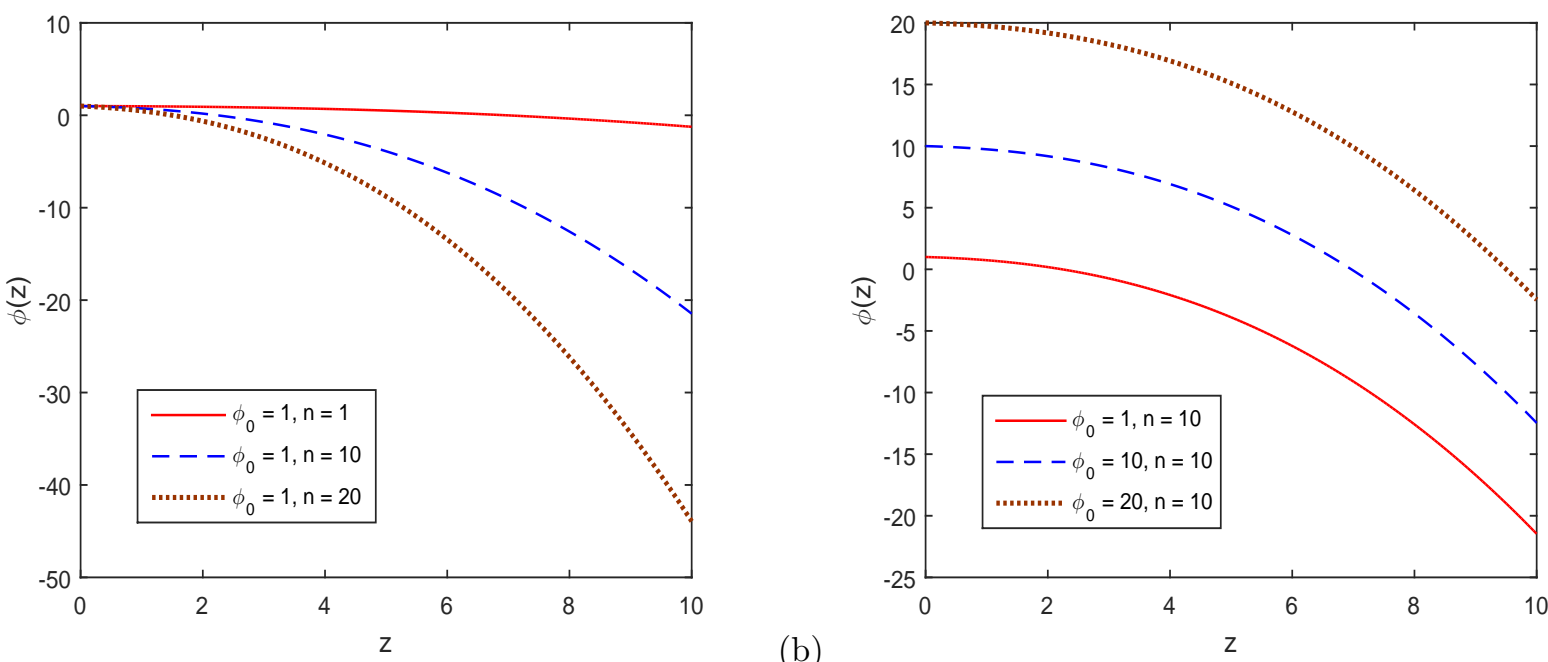

Figure 2: The plots of scalar field $\phi(z)$ versus redshift $z$ for $H_{0}=71.27$.

Using Eqs. (30) and (31) in Eqs. (22) - (25), we obtain the expressions for the trace $T$, energy density $\rho$ of the fluid, and EoS parameter $\omega$ as

$$
\begin{gathered}
T=-9 k^{2}(1+z)+\frac{9 \alpha k^{4}}{2}(1+z)^{2}\left[31+(1+z)^{\frac{1}{2}}-\frac{14 k^{2}}{1+z}\right] \\
\rho=3 k^{2}(1+z)-\frac{9 \alpha k^{4}}{2}(1+z)(22+23 z)-\frac{n^{2}}{2}(1+z)^{6} \\
\omega=-1+\frac{1-\frac{n^{2}}{k^{2}}(1+z)^{5}-\frac{3}{2} \alpha k^{2}(1+z)\left[2(1+2 z)+\frac{8}{1+z}-\frac{k}{(1+z)^{\frac{1}{2}}}+16\right]}{3-\frac{9 \alpha k^{2}}{2}(22+23 z)-\frac{n^{2}}{2 k^{2}}(1+z)^{5}}
\end{gathered}
$$

Eq. (32) represents the expression for trace of the total energy momentum tensor $T_{i j}$ and Figure 3 represents the plot of the trace $T$ over the variation of redshift $z$ for the different values of $\alpha$ and constrained by the Hubble constant $H_{0}$. At present $z=0, T_{0}=-9 k^{2}+144 \alpha k^{4}-63 \alpha k^{6}$ which depends on the value of $k$ and $\alpha$.

Eq. (33) represents the expression for energy density $\rho$ in terms of redshift $z$ and Figures 4(a), 4(b), and 4(c) show the variation of energy density $\rho(z)$ over the variation of redshift $z$ constrained by Hubble constant $H_{0}$ for different choices of the value of $\alpha$ and $n$. At present $z=0, \rho_{0}=3 k^{2}\left(1-33 \alpha k^{2}\right)-\frac{n^{2}}{2}$ and in past as $z$ increases, $\rho$ increases to a large value. Here, $\rho_{0}>0$ for $3 k^{2}\left(1-33 \alpha k^{2}\right)>\frac{n^{2}}{2}$.

Eq. (34) denotes the expression for EoS parameter $\omega(z)$ and Figure 5(a), 5(b), and 5(c) represent the evolution of EoS parameter $\omega$ over the variation of redshift $z$ constrained by $H_{0}$. One can see that at present $z=0$,

$$
\omega_{0}=-1+\frac{2 k^{2}-78 \alpha k^{4}+3 \alpha k^{5}-2 n^{2}}{6 k^{2}-198 \alpha k^{4}-n^{2}}
$$

and $\lim _{z \rightarrow \infty} \omega(z)=1$ which are consistent with recent observations [1]-[7]. 


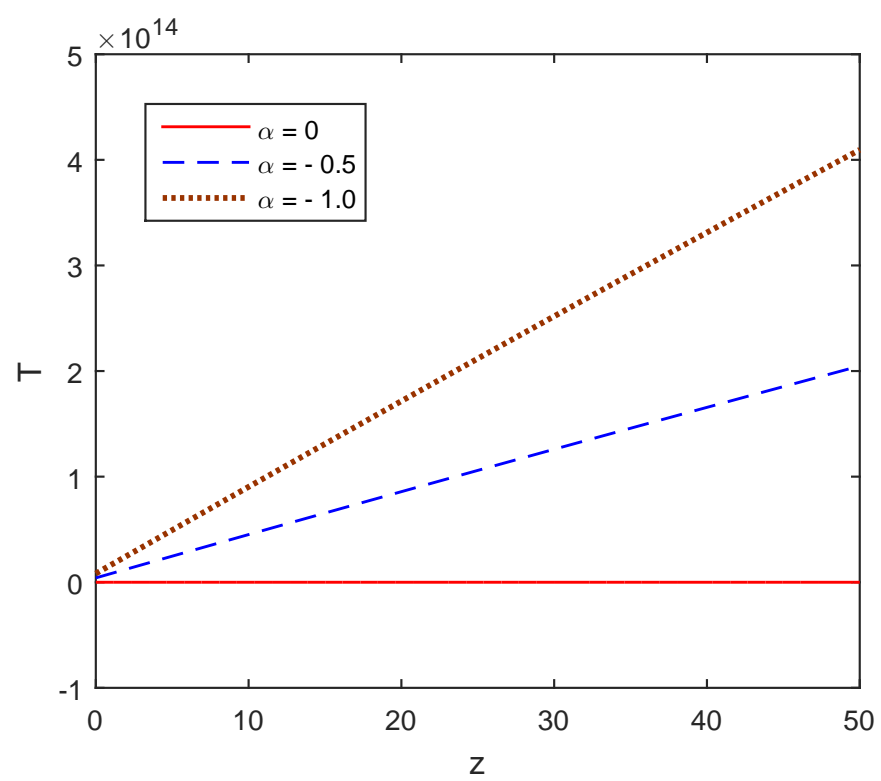

Figure 3: The plots of trace $T(z)$ versus redshift $z$ for $H_{0}=71.27$.

(a)

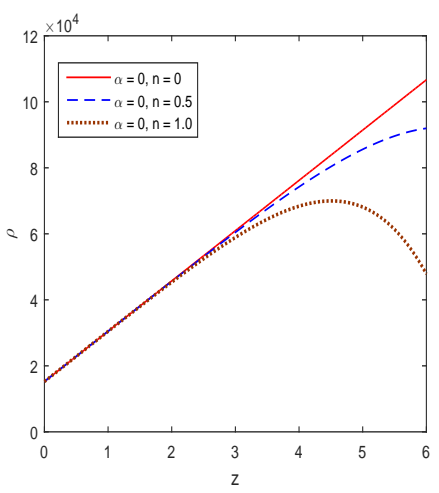

(b)

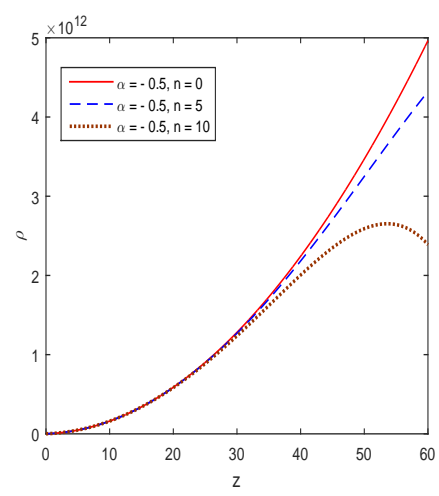

(c)

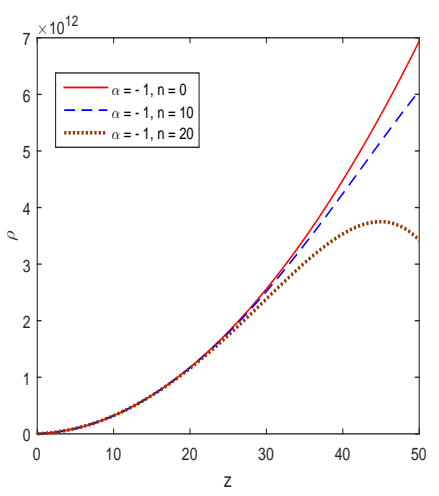

Figure 4: The plots of energy density $\rho(z)$ versus redshift $z$ for $H_{0}=71.27$.

(a)

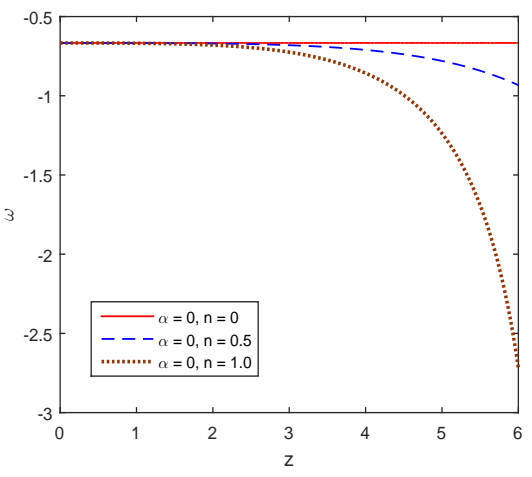

(b)

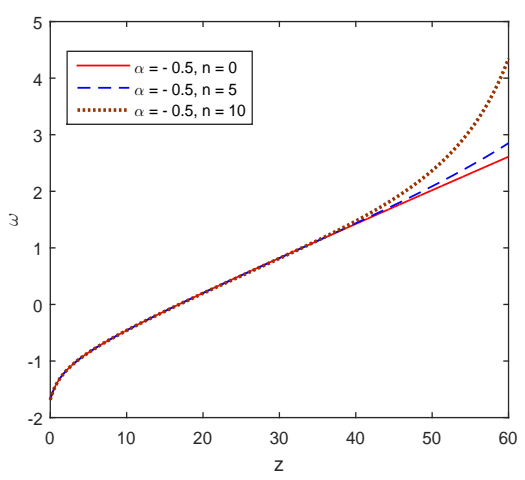

(c)

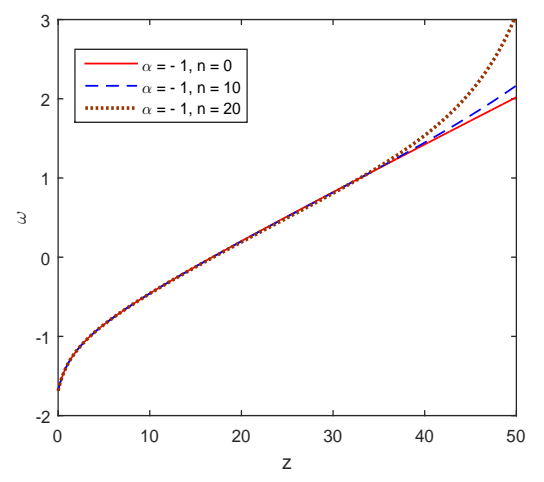

Figure 5: The plots of EoS parameter $\omega(z)$ versus redshift $z$ for $H_{0}=71.27$. 


\section{Age of the Universe}

The age of the cosmic universe is calculated as

$$
\begin{gathered}
t_{0}-t=\int_{0}^{z} \frac{d z}{(1+z) H(z)} \\
H_{0}\left(t_{0}-t\right)=2\left[1-\frac{1}{\sqrt{1+z}}\right]
\end{gathered}
$$

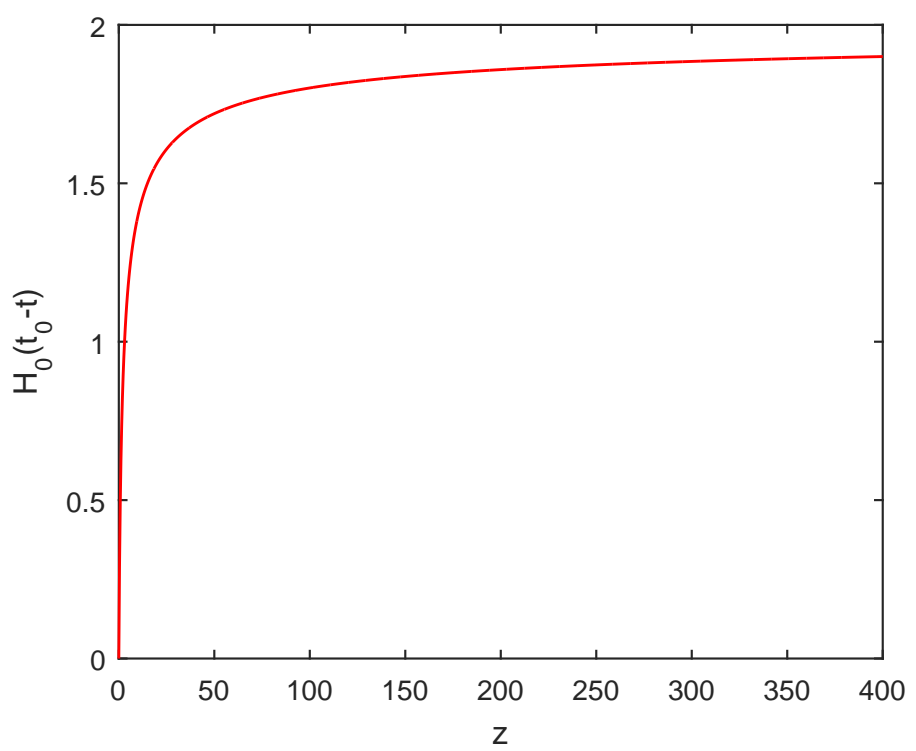

Figure 6: The plot of cosmic time $t$ versus redshift $z$

From Figure 6 , the constant graph of time shows the age of the universe i.e. $\lim _{z \rightarrow \infty} H_{0}\left(t_{0}-t\right)=2 \Longrightarrow H_{0} t_{0}=2$ and hence, the present age of the universe $t_{0}=27.44$ Gyrs.

\subsection{Transit Model: Case-II: when $\dot{H}=\frac{c_{1}}{a^{4}}$}

From equations $\dot{H}=\frac{c_{1}}{a^{4}}$ and $\dot{H}=-(1+z) H \frac{d H}{d z}$ we have obtained the Hubble parameter $H$ as

$$
H=\left[2 c_{2}-\frac{c_{1}}{2}(1+z)^{4}\right]^{\frac{1}{2}}
$$

where $c_{1}$ and $c_{2}$ are arbitrary constants. At present $z=0$, we found the $H_{0}=\left[2 c_{2}-\frac{c_{1}}{2}\right]^{\frac{1}{2}}$. Figure 7 represents its variation over redshift $z$.

Now, we have

$$
\frac{\dot{a}}{a}=\sqrt{2 c_{2}-\frac{c_{1}}{2 a^{4}}}
$$

Let us choose the arbitrary constants $c_{1}, c_{2}$ as $c_{2}=-c_{1}=m_{1}$ and $c_{2}=c_{1}=m_{1}$, in these cases, integrating Eq. (38), we get the scale factor as

$$
a(t)=\frac{1}{\sqrt{2}}\left[\sinh \left(2 \sqrt{2} m_{1} t+4 m_{2}\right)\right]^{\frac{1}{2}}
$$

and

$$
a(t)=\frac{1}{\sqrt{2}}\left[\cosh \left(2 \sqrt{2} m_{1} t+4 m_{2}\right)\right]^{\frac{1}{2}}
$$


respectively. Where $m_{1}, m_{2}$ are integrating constants. Here, we have considered first case.

Now, we have calculated the deceleration parameter $q(z)$ as

$$
q=\frac{d}{d t}\left(\frac{1}{H}\right)-1 \quad \Longrightarrow \quad q(z)=-\frac{4 c_{2}+c_{1}(1+z)^{4}}{4 c_{2}-c_{1}(1+z)^{4}}
$$

At present $z=0, q_{0}=-\frac{4 c_{2}+c_{1}}{4 c_{2}-c_{1}}$ and in the past as $z \rightarrow \infty$, we have $\lim _{z \rightarrow \infty} q(z)=1$. Also, Figure 7 (b) represents the variation of deceleration parameter $q(z)$ over the variation of redshift $z$. One can see that $q(z)$ is an increasing function of redshift $z$ and at present $z=0, q_{0}=-0.6$ and at $z_{t}=0.414$ expansion transits its phase from deceleration to acceleration. Thus, our universe model is a transit phase model.
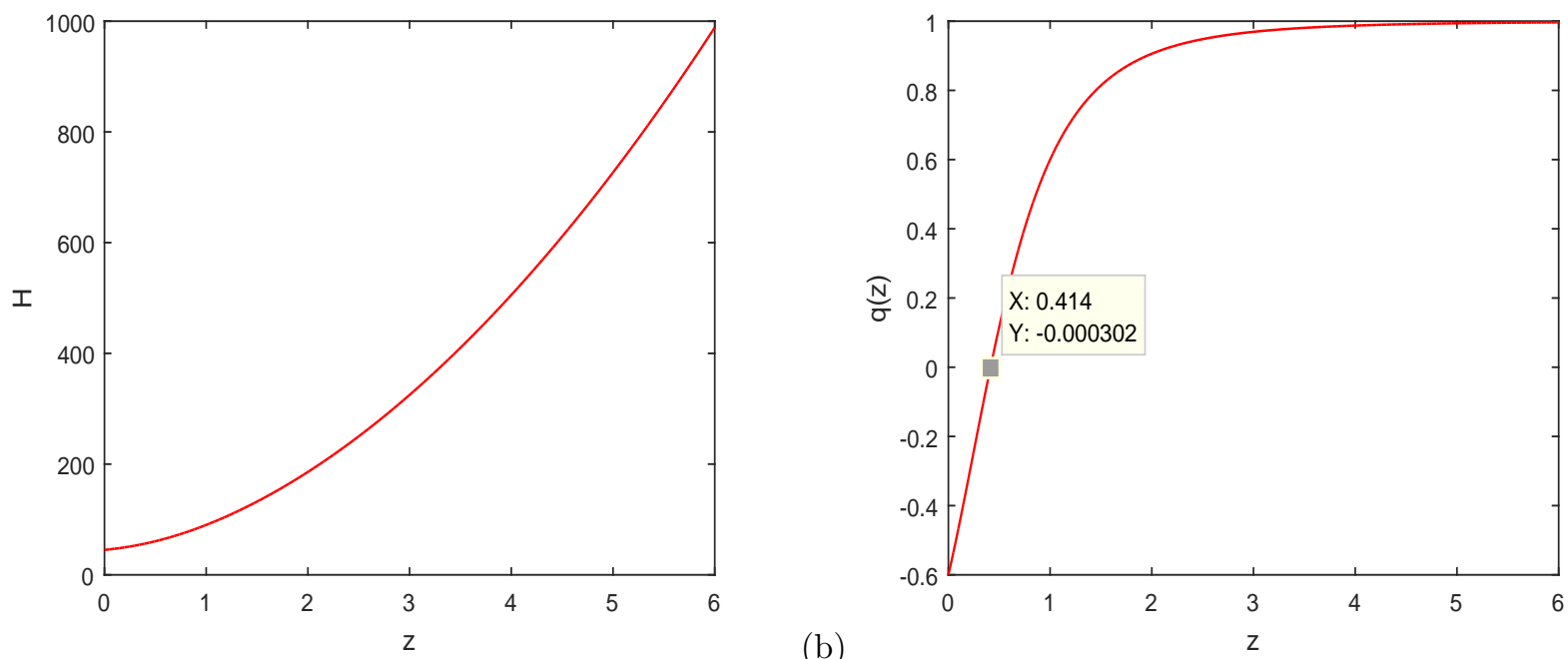

(a)

Figure 7: (a)The plot of Hubble parameter $H$ versus redshift $z$ and (b) The plot of deceleration parameter $q(z)$ versus redshift $z$ for $c_{1}=-\frac{2}{5} H_{0}^{2}, c_{2}=\frac{2}{5} H_{0}^{2}$.

Now, using Eq. (37) in Eqs. (22) - (25), we obtain trace $T$, energy density $\rho$ and EoS parameter $\omega$ in terms of redshift $z$ as

$$
\begin{gathered}
T=-24 c_{2}-\frac{36 \alpha c_{1}^{2}(1+z)^{8}}{\left[2 c_{2}-\frac{c_{1}}{2}(1+z)^{4}\right]}\left[3-2 c_{2}-3 c_{1}(1+z)^{4}+c_{1}(1+z)^{4}\left[2 c_{2}-\frac{c_{1}}{2}(1+z)^{4}\right]^{\frac{1}{2}}\right] \\
\rho=6 c_{2}-\frac{3 c_{1}}{2}(1+z)^{4}+36 \alpha c_{1}(1+z)^{4}\left[2 c_{2}+c_{1}(1+z)^{3}-2 c_{1}(1+z)^{4}\right]-\frac{n^{2}}{2}(1+z)^{6} \\
\omega=-1+\frac{L(z)}{\rho(z)}
\end{gathered}
$$

where

$$
\begin{gathered}
L(z)=-2 c_{1}(1+z)^{4}+\frac{12 \alpha c_{1}(1+z)^{3}}{\left[2 c_{2}-\frac{c_{1}}{2}(1+z)^{4}\right]} \times \\
{\left[4 c_{2}\left(5-6 c_{2}\right)(1+z)+24 c_{1} c_{2}(1+z)^{5}-2 c_{1}^{2}(1+z)^{8}+\frac{3 c_{1}^{2}}{2}(1+z)^{9}+\left[30 c_{2}+\frac{5}{2} c_{1}(1+z)^{4}-c_{1}^{2}(1+z)^{9}\right]\left[2 c_{2}-\frac{c_{1}}{2}(1+z)^{4}\right]^{\frac{1}{2}}\right]-n^{2}(1+z)^{6}}
\end{gathered}
$$

Eq. (42) represents the expression for trace $T(z)$ of the total energy momentum tensor $T_{i j}$ and Figure 8 represents the plot of the trace $T$ over the variation of redshift $z$ for the different values of $\alpha$ and constrained by the Hubble constant $H_{0}$. At present $z=0, T_{0}=-24 c_{2}-\frac{72 \alpha c_{1}^{2}}{4 c_{2}-c_{1}}\left[3\left(1-c_{1}\right)-2 c_{2}+c_{1}\left(2 c_{2}-\frac{c_{1}}{2}\right)^{\frac{1}{2}}\right]$ which depends on the value of $c_{1}, c_{2}$ and $\alpha$.

Eq. (43) represents the expression for energy density $\rho(z)$ in terms of redshift $z$ and Figures $9(\mathrm{a}), 9(\mathrm{~b})$, and $9(\mathrm{c})$ show the variation of energy density $\rho(z)$ over the variation of redshift $z$ constrained by Hubble constant $H_{0}$ for different choices of the value of $\alpha$ and $n$. At present $z=0, \rho_{0}=6 c_{2}-\frac{3}{2} c_{1}+36 \alpha c_{1}\left(2 c_{2}-c_{1}\right)-\frac{n^{2}}{2}$ and in past as $z$ increases, $\rho$ increases to a large value. 
Eq. (44) denotes the expression for EoS parameter $\omega(z)$ and Figures 10(a), 10(b), and 10(c) represent the evolution of EoS parameter $\omega$ over the variation of redshift $z$ constrained by $H_{0}$. One can see that at present $z=0$,

$$
\omega_{0}=-1+\frac{-2 c_{1}+\frac{24 \alpha c_{1}}{4 c_{2}-c_{1}}\left[4 c_{2}\left(5-6 c_{2}\right)+24 c_{1} c_{2}-\frac{1}{2} c_{1}^{2}+\left(30 c_{2}+\frac{5}{2} c_{1}-c_{1}^{2}\right)\left(2 c_{2}-\frac{1}{2} c_{1}\right)^{\frac{1}{2}}\right]-n^{2}}{6 c_{2}-\frac{3}{2} c_{1}+36 \alpha c_{1}\left(2 c_{2}-c_{1}\right)-\frac{n^{2}}{2}}
$$

and $\lim _{z \rightarrow \infty} \omega(z)=\infty$ which are consistent with recent observations [1]-[7].

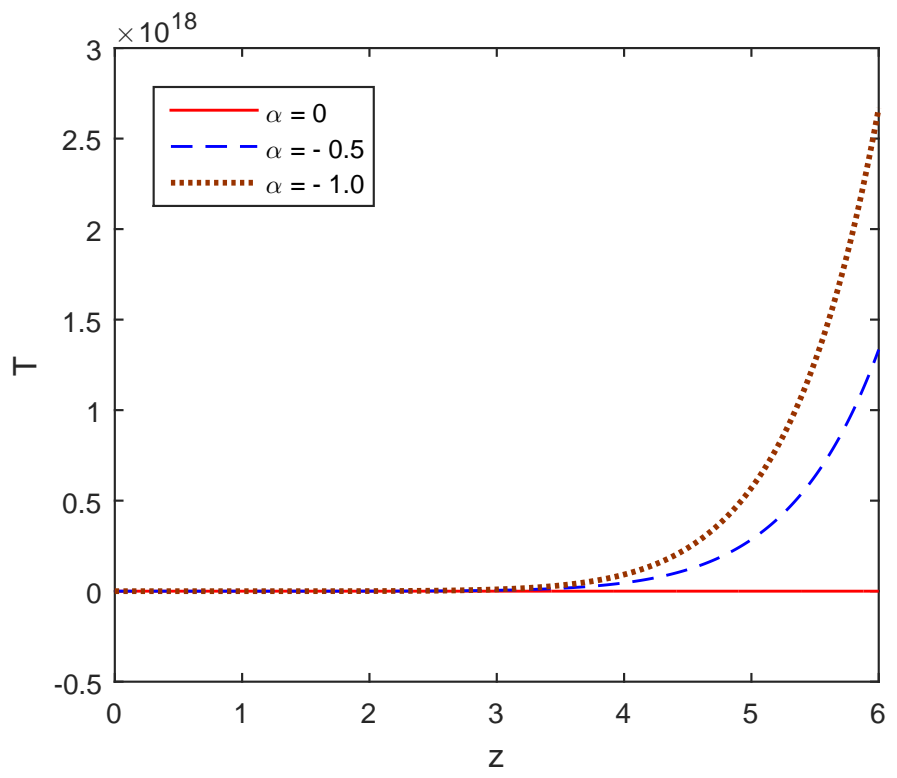

Figure 8: The plots of trace $T(z)$ versus redshift $z$ for $H_{0}=71.27$.

(a)

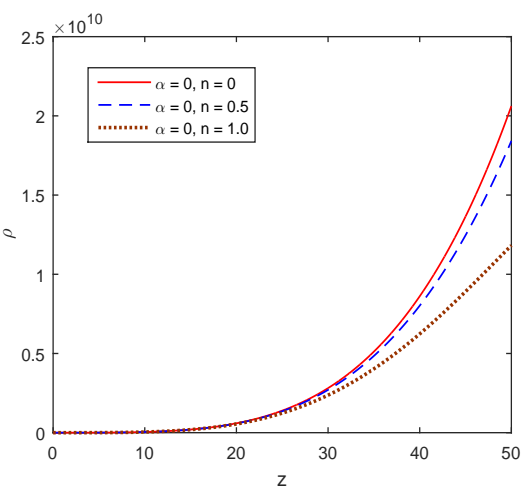

(b)

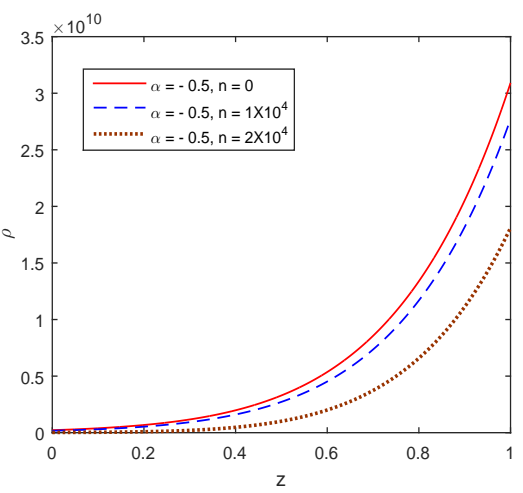

(c)

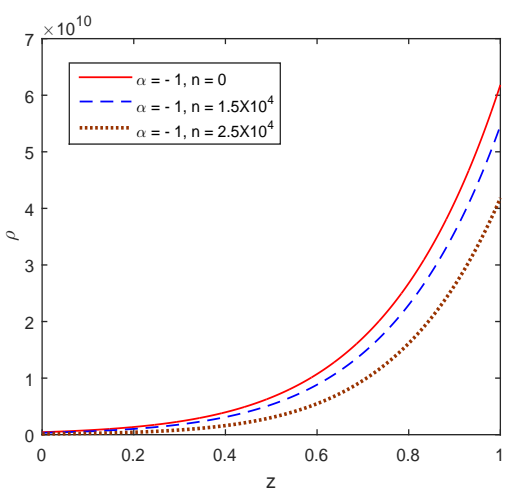

Figure 9: The plots of energy density $\rho(z)$ versus redshift $z$ for $H_{0}=71.27$. 
(a)

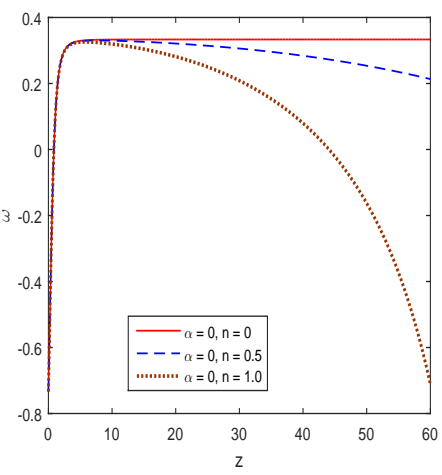

(b)

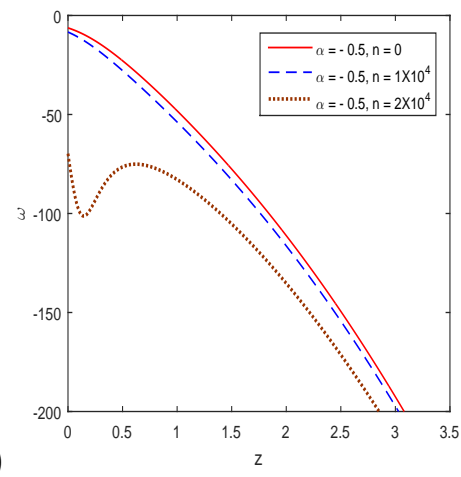

(c)

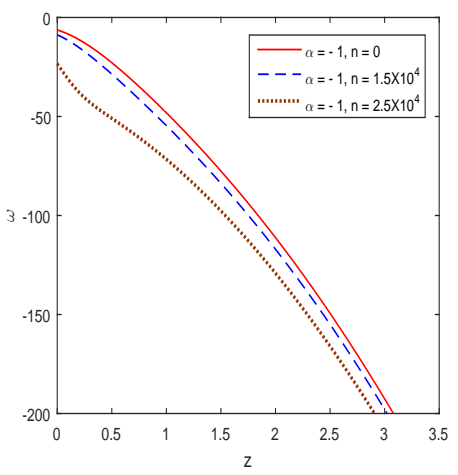

Figure 10: The plots of EoS parameter $\omega(z)$ versus redshift $z$ for $H_{0}=71.27$.

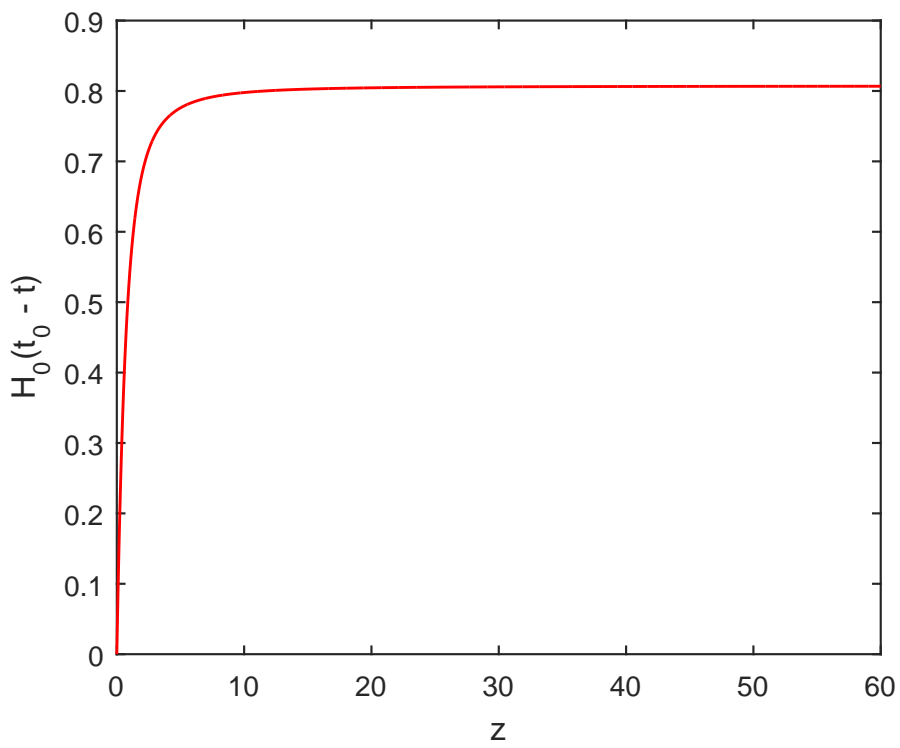

Figure 11: The plot of cosmic time $t$ versus redshift $z$

\section{Age of the Universe}

The age of the cosmic universe is calculated as

$$
\begin{gathered}
t_{0}-t=\int_{0}^{z} \frac{d z}{(1+z) H(z)} \\
\left(t_{0}-t\right)=\frac{1}{4} \sqrt{\frac{2}{c_{2}}}\left[\tanh ^{-1}\left(\frac{4 c_{2}-c_{1}}{4 c_{2}}\right)^{\frac{1}{2}}-\tanh ^{-1}\left(\frac{4 c_{2}-c_{1}(1+z)^{4}}{4 c_{2}}\right)^{\frac{1}{2}}\right]
\end{gathered}
$$

From Figure 11, the constant graph of time shows the age of the universe i.e. $\lim _{z \rightarrow \infty} H_{0}\left(t_{0}-t\right)=0.8233 \Longrightarrow H_{0} t_{0}=0.8233$ and hence, the present age of the universe $t_{0}=11.3$ Gyrs .

\section{Concluding Remarks}

In this paper, we have investigated a flat FRW cosmological model filled with perfect fluid coupled with the zero-mass scalar field in the higher derivative theory of gravity. We have obtained two types universe models, first one is accelerating universe (power-law cosmology) and second one is transit phase model (hyperbolic expansion-law). The main features of the models 
are as:

- The scale-factor $a(t)$ is obtained from the field equations without assumptions.

- These models evolves with high redshift values $0 \leq z<\infty$.

- For the transit phase model the deceleration parameter shows signature-flipping with transition value $z_{t}=0.414$ which is consistent with recent observations. The present value deceleration parameter is obtained as $q_{0}=-0.6$ for transit model and $q_{0}=-0.5$ for accelerating universe.

- The EoS parameter $\omega$ varies from positive to negative values with the evolution the universe, which reveals the formation of different structures during the evolution of the universe.

- At $t=0$, the scale factor $a(t)$ has a finite value.

- The present age of the universe for transit phase model is obtained as 11.3Gyrs.

\section{Acknowledgment}

One of the authors (DM) is thankful to IASE (Deemed to be University), Sardarshahar, Rajsthan, India for providing facilities and support where part of this work is carried out.

\section{References}

[1] P. M. Garnavich et al., Constraints on cosmological models from Hubble space telescope observations of high Z supernovae, Astrophys. J. 493 (1998) L53-L57.

[2] P. M. Garnavich et al., Supernova limits on the cosmic equation of state, Astrophys. J. 509 (1998) 74-79.

[3] S. Perlmutter et al., Measurements of the cosmological parameters Omega and Lambda from the first 7 supernovae at $z \geq 0.35$, Astrophys. J. 483 (1997) 565-581.

[4] S. Perlmutter et al., Discovery of a supernova explosion at half the age of the universe and its cosmological implications, Nature 391 (1998) 51-54.

[5] S. Perlmutter et al., Measurements of omega and lambda from 42 high-redshift supernovae, Astrophys. J. 517 (1999) 565-586.

[6] A. G. Riess et al., Observational evidence from supernovae for an accelerating universe and a cosmological constant, Astron. J. 116 (1998) 1009-1038.

[7] B. P. Schmidt et al., The high-Z supernova search: Measuring cosmic deceleration and global curvature of the universe using type IA supernovae, Astrophys. J. 507 (1998) 46-63.

[8] A. A. Starobinsky, A New Type of Isotropic Cosmological Models Without Singularity, Phys. Lett. B, 91 (1980) 99-102.

[9] A. H. Guth, Inflationary universe: A possible solution to the horizon and flatness problems, Phys. Rev. D 23 (1981) $347-356$.

[10] K. S. Stelle, Renormalization of higher-derivative quantum gravity, Phys. Rev. D 16 (1977) 953-969.

[11] A. D. Linde, Particle Physics and Cosmology. Gordon and Breach, New York (1990).

[12] E. Kolb and M. S. Turner, The early Universe. Addison-Wesley, New York (1990).

[13] L. P. Chimento and A. S. Jakubi, Exact Solutions and Scalar Fields in Gravity, Int. J. Mod. Phys. D 5 (1996) 71.

[14] I. Muller, Zum Paradoxon der Wrmeleitungstheorie, Z. Phys. 198 (1967) 329-344.

[15] W. A. Hiscock and J. Salmonson, Dissipative Boltzmann-Robertson-Walker cosmologies, Phys. Rev. D 43 (1991) 32493258 . 
[16] W. Israel and J. M. Stewart, Nonstationary irreversible thermodynamics: A causal relativistic theory, Ann. Phys. (N.Y.) 100 (1976) 310-331.

[17] D. Pavon, D. Jou and J. Casas-Vazquez, On a covariant formulation of dissipative phenomena, Ann. Inst. Henri Poincare, A. Phys. Theor. 36 (1982) 79-88.

[18] D. Pavon, Nonequilibrium fluctuations in cosmic vacuum decay, Phys. Rev. D 43 (1991) 375-378.

[19] B. Ratra and P. J. E. Peebles, Cosmological consequences of a rolling homogeneous scalar field, Phys. Rev. D 37 (1988) 3406-3427.

[20] D. Pavon and I. W. Zimdahl, Dark matter and dissipation, Phys. Lett. A. 179 (1993) 261-265.

[21] I. W. Zimdahl et al., Cosmic antifriction and accelerated expansion, Phys. Rev. D 64 (2001) 063501.

[22] B. Ram, Infinitely many solutions of Einstein cosmology in slow-roll, Phys. Lett. A 172 (1993) 404-406.

[23] G. F. R. Ellis and M. S. Madsen, Exact scalar field cosmologies, Class. Quantum Gravity 8 (1991) 667-676.

[24] J. D. Barrow and P. Saich, Scalar-field cosmologies, Class. Quantum Gravity 10 (1993) 279-283.

[25] J. D. Barrow and J. P. Mimoso, Perfect fluid scalar-tensor cosmologies, Phys. Rev. D 50 (1994) 3746-3754.

[26] J. P. Mimoso and D. Wands, Massless fields in scalar-tensor cosmologies, Phys. Rev. D 51 (1995) 477-489.

[27] R. Casana, C. A. M. de Melo and B. M. Pimentel, Massless DKP field in a Lyra manifold, Class. Quantum Gravity 24 (2007) 723-736.

[28] N. I. Singh, S. S. Singh and S. R. Devi, Casual viscous universe coupled with zero-mass scalar field in higher derivative theory, Astrophys. Space Sci. 334 (2011) 187-191.

[29] D. C. Maurya and R. Zia, Brans-Dicke Scalar Field Cosmological Model in Lyra's Geometry, Phys. Rev. D 100 (2019) 023503.

[30] A. R. Roy and M. N. Verma, R-W cosmological models with zero-mass scalar fields for different equation of state, Astrophys. Space Sci. 138 (1987) 217-219.

[31] D. R. K. Reddy, R. Venkateswarlu, Bianchi type-I universe in the presence of zero-mass scalar fields, Astrophys. Space Sci. 136 (1987) 17-20.

[32] A. Pradhan, K. L. Tiwari and A. Beesham, LRS Bianchi I cosmological models in the presence of zero-mass scalar field, Indian J. pure appl. Math. 32 (2001) 789-795.

[33] R. Venkateswarlu and K. P. Kumar, Plane symmetric string cosmological models in zero-mass scalar field, Int. J. Theor. Phys. 49 (2010) 1894-1898. 